\title{
ESTRUTURA E PROPRIEDADES DE ELIPTICINAS
}

\author{
José J. V. Cirino, Paulo Belletato e Sócrates de O. Dantas \\ Departamento de Física, Instituto de Ciências Exatas, Universidade Federal de Juiz de Fora, 36036-330 Juiz de Fora-MG \\ Lucicleide Ribeiro, Gilson R. Ferreira e Hélio F. dos Santos* \\ Departamento de Química, Instituto de Ciências Exatas, Universidade Federal de Juiz de Fora, 36036-330 Juiz de Fora-MG
}

Recebido em 9/12/03; aceito em 1/9/04; publicado na web em 12/11/04

\begin{abstract}
STRUCTURE AND PROPERTIES OF ELLIPTICINES. The ellipticines constitute a broad class of molecules with antitumor activity. In the present work we analyzed the structure and properties of a series of ellipticine derivatives in the gas phase and in solution using quantum mechanical and Monte Carlo methods. The results showed a good correlation between the solvation energies in water obtained with the continuum model and the Monte Carlo simulation. Molecular descriptors were considered in the development of QSAR models using the DNA association constant $\left(\log \mathrm{K}_{\text {app }}\right)$ as biological data. The results showed that the DNA binding is dominated by electronic parameters, with small contributions from the molecular volume and area.
\end{abstract}

Keywords: ellipticine; $a b$ initio calculation; QSAR.

\section{INTRODUÇÃO}

Elipticinas (Figura 1) constituem uma ampla classe de compostos com atividade antitumoral ${ }^{1}$. Além da alta citotoxicidade em células cancerosas, estas moléculas são especialmente interessantes do ponto de vista clínico devido aos efeitos colaterais reduzidos ${ }^{2}$. O modo de ação das elipticinas tem sido amplamente estudado, entretanto, conclusões definitivas sobre os aspectos moleculares relevantes para a ação biológica não foram ainda relatadas ${ }^{3}$. O núcleo molecular básico das elipticinas é constituído de um grupamento carbazol ligado a um anel piridina, propiciando a deslocalização eletrônica sobre toda a molécula. Esta estrutura relativamente simples tem permitido a síntese de mais de 70 análogos distintos ${ }^{3}$, com as principais modificações sendo a inclusão de um grupo hidroxila nas posições $\mathrm{C}_{9}$ e $\mathrm{C}_{7}$, e alquilação das posições $\mathrm{N}_{2}, \mathrm{~N}_{6}$ e $\mathrm{C}_{1}$ (ver Figura 1). Mais recentemente ${ }^{4}$, novas elipticinas substituídas em $C_{9}$ foram sintetizadas visando obter derivados com citotoxicidade seletiva.

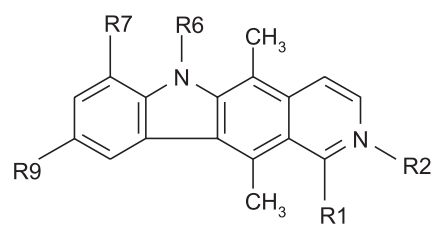

Figura 1. Estrutura básica das elipticinas

No presente trabalho, a estrutura e as propriedades moleculares de algumas elipticinas foram determinadas através de cálculos $a b$ initio em fase gasosa. As energias de solvatação foram calculadas utilizando modelos contínuos de solvatação e simulação de Monte Carlo. A estrutura do solvente ao redor de alguns sítios das moléculas foi analisada visando identificar características do processo de solvatação, específicas para os diferentes análogos. Na parte final do trabalho, modelos quantitativos de relação estrutura-atividade (QSAR) foram construídos e analisados.

\footnotetext{
*e-mail: helius@quimica.ufjf.br
}

\section{METODOLOGIA DE CÁLCULO}

O conjunto de moléculas estudadas no presente trabalho é apresentado na Tabela 1. Estes análogos foram selecionados de acordo com a disponibilidade de parâmetros biológicos quantitativos ${ }^{3}$ e características estruturais dissimilares dentro desta classe de moléculas. Para cada composto descrito na Tabela 1, a geometria foi completamente otimizada no nível HF/6-31G(d) e caracterizada como mínimo verdadeiro na Superfície de Energia Potencial (PES) através do cálculo de frequiências harmônicas. Cargas atômicas ChelpG (Q), momento de dipolo elétrico $(\mu)$ e energias dos orbitais moleculares de fronteira (HOMO e LUMO) foram calculadas no mesmo nível de teoria, utilizando o programa Gaussian/985. A área superficial (A) e o volume molecular (V) foram obtidos no programa Spartan-Pro ${ }^{6}$ utilizando as geometrias otimizadas no nível ab initio de teoria. Energias de solvatação em água $(\varepsilon=78,39)$ e $\mathrm{CCl}_{4}(\varepsilon=2,24)$ foram calculadas dentro do formalismo da mecânica quântica utilizando o modelo contínuo PCM-UAHF ("Polarized Continum Model-United Atoms HartreeFock" $)^{7,8}$.

As geometrias otimizadas no nível HF/6-31G(d) foram consideradas nas simulações de Monte Carlo realizadas com o programa DIADORIM ${ }^{9}$. Os sistemas estudados foram representados por caixas de simulação de formato cúbico contendo 2000 moléculas de solvente $\left(\mathrm{H}_{2} \mathrm{O}\right.$ ou $\left.\mathrm{CCl}_{4}\right)$ e 1 molécula de soluto. Condições usuais em simulação de líquidos foram empregadas, ou seja, condições periódicas de contorno, convenção de imagem mínima, raio de corte esférico e amostragem preferencial próxima ao soluto ${ }^{10}$. Para o solvente água, o modelo utilizado foi o TIP4P ${ }^{11,12}$ e para o $\mathrm{CCl}_{4}$ foi construído um modelo a partir de sua geometria $a b$ initio (HF/6-31G(d)), dos parâmetros de Lennard-Jones do campo de força OPLS ${ }^{13}$ e das cargas parciais ChelpG obtidas nos cálculos ab initio. Esse modelo foi submetido a um teste em que foram calculados alguns parâmetros físico-químicos da substância pura. Para a água pura obtivemos a densidade igual a $1,004 \mathrm{~g} \mathrm{~cm}^{-3}$ (valor experimental: $0,997 \mathrm{~g} \mathrm{~cm}^{-3}$ ) e calor de vaporização de $10,70 \mathrm{kcal} \mathrm{mol}^{-1}$ (valor experimental: $10,52 \mathrm{kcal} \mathrm{mol}^{-1}$ ), enquanto que para o $\mathrm{CCl}_{4}$ esses valores foram 1,574 $\mathrm{g} \mathrm{cm}^{-3}$ (valor experimental: $1,594 \mathrm{~g} \mathrm{~cm}^{-3}$ ) e 7,76 kcal mol-1 (valor experimental: 7,76 kcal mol-1), respectivamente. 
Tabela 1. Derivados da elipticina considerados no presente trabalho

\begin{tabular}{|c|c|c|c|c|c|c|c|}
\hline & Carga & & $R \boldsymbol{R}$ & $R 2$ & $R 6$ & $R 7$ & $R 9$ \\
\hline Elipticina & 0 & E01 & $\mathrm{H}$ & - & $\mathrm{H}$ & $\mathrm{H}$ & $\mathrm{H}$ \\
\hline 9-amino-elipticina & 0 & E02 & $\mathrm{H}$ & - & $\mathrm{H}$ & $\mathrm{H}$ & $\mathrm{NH}_{2}$ \\
\hline 9-hidroxi-elipticina & 0 & E03 & $\mathrm{H}$ & - & $\mathrm{H}$ & $\mathrm{H}$ & $\mathrm{OH}^{2}$ \\
\hline 7-hidroxi-elipticina & 0 & E04 & $\mathrm{H}$ & - & $\mathrm{H}$ & $\mathrm{OH}$ & $\mathrm{H}$ \\
\hline 9-metoxi-elipticina & 0 & E05 & $\mathrm{H}$ & - & $\mathrm{H}$ & $\mathrm{H}$ & $\mathrm{OCH}_{3}$ \\
\hline Elipticinium & +1 & E06 & $\mathrm{H}$ & $\mathrm{CH}_{3}$ & $\mathrm{H}$ & $\mathrm{H}$ & $\mathrm{H}^{3}$ \\
\hline 2N-metil-9-hidroxi-elipticinium & +1 & E07 & $\mathrm{H}$ & $\mathrm{CH}_{3}{ }^{3}$ & $\mathrm{H}$ & $\mathrm{H}$ & $\mathrm{OH}$ \\
\hline 2N-metil-1-metil-elipticinium & +1 & E08 & $\mathrm{CH}_{3}$ & $\mathrm{CH}_{3}$ & $\mathrm{H}$ & $\mathrm{H}$ & $\mathrm{H}$ \\
\hline 2N-metil-6N-metil-elipticinium & +1 & E09 & $\mathrm{H}^{3}$ & $\mathrm{CH}_{3}^{3}$ & $\mathrm{CH}_{3}$ & $\mathrm{H}$ & $\mathrm{H}$ \\
\hline 2N-metil-6N-metil-9-hidroxi-elipticinium & +1 & E10 & $\mathrm{H}$ & $\mathrm{CH}_{3}^{3}$ & $\mathrm{CH}_{3}^{3}$ & $\mathrm{H}$ & $\mathrm{OH}$ \\
\hline
\end{tabular}

$\mathrm{O}$ cálculo de $\Delta \mathrm{E}_{\mathrm{SOLV}}$ com o método de Monte Carlo foi feito a partir da energia configuracional do sistema, que é obtida por meio da função de potencial intermolecular entre duas moléculas a e b, descrita pela soma dos potenciais de Coulomb e de Lennard-Jones (Equações 1 e 2):

$$
\begin{aligned}
& E\left(r_{i j}\right)=q_{i} q_{j} / r_{i j}+4 \varepsilon_{i j}\left[\left(\sigma_{i j} / r_{i j}\right)^{12}-\left(\sigma_{i j} / r_{i j}\right)^{6}\right] \\
& E_{a b}=\sum_{i}^{a} \sum_{j}^{b} E\left(r_{i j}\right)
\end{aligned}
$$

em que $r_{i j}$ é a distância entre o sítio $i$ na molécula a e o sítio $j$ na molécula b, e $q_{k}$ são as cargas parciais nos sítios atômicos. Os parâmetros $\sigma_{i j}$ e $\varepsilon_{i j}$ das interações não diagonais podem ser obtidos pelas regras de combinação $\varepsilon_{i j}=\left(\varepsilon_{i} \varepsilon_{j}\right)^{1 / 2}$ e $\sigma_{i j}=\left(\sigma_{i} \sigma_{j}\right)^{1 / 2}$. Desta forma, é possível calcular a energia total do sistema e também as energias de interação solvente-solvente e soluto-solvente $\left(\Delta \mathrm{E}_{\mathrm{SOLV}}\right)$. Para equilibrar as caixas de simulação com os solventes água e $\mathrm{CCl}_{4}$ foram geradas $5 \times 10^{6}$ configurações. Uma vez equilibradas as caixas de simulação, procedeu-se ao cálculo dos valores médios das propriedades termodinâmicas e estruturais com a geração de mais $10 \times 10^{6}$ configurações para cada sistema. $\mathrm{O}$ raio de corte para as interações água-água foi de $8,5 \AA$ e para as interações soluto-solvente foi de $15 \AA$. No processo de geração de novas configurações em água, os movimentos de translação e rotação do solvente foram, respectivamente, $0,15 \AA$ e 15 graus. Em $\mathrm{CCl}_{4}$, o raio de corte para as interações $\mathrm{CCl}_{4}-\mathrm{CCl}_{4}$ foi de $25 \AA$, e para as interações soluto-solvente foi de $15 \AA$. No processo de geração de novas configurações em $\mathrm{CCl}_{4}$, os movimentos de translação e rotação do solvente foram, respectivamente, $0,15 \AA$ e 15 graus. As simulações em ambos os solventes foram realizadas no "ensemble" $\mathrm{NpT}$, a $25^{\circ} \mathrm{C}$ e 1 atm.

Na última parte do trabalho, Correlação Linear Múltipla foi utilizada na busca de modelos quantitativos de relação estrutura-atividade (QSAR) utilizando os descritores eletrônicos quanto-mecâni$\cos \left(\mathrm{Q}_{\mathrm{i}}, \mu, \mathrm{E}_{\text {номо }}\right.$ e $\left.\mathrm{E}_{\text {LUMO }}\right)$, parâmetros estéreos (A e V) e energias de solvatação em água e $\mathrm{CCl}_{4}$. Esta análise foi feita utilizando o programa BuildQSAR ${ }^{14}$.

\section{RESULTADOS E DISCUSSÃO}

\section{Características estruturais das elipticinas}

Os principais parâmetros geométricos calculados a partir das geometrias otimizadas no nível HF/6-31G(d) para os análogos elipticina (E01) e 9-metoxi-elipticina (E05) foram comparados com os dados obtidos no estado sólido através de experimentos de difração de raios $\mathrm{X}^{15,16}$. Os resultados mostraram desvios menores que $0,05 \AA$ e $2^{\circ}$, os quais podem ser atribuídos às forças intermoleculares pre- sentes na rede cristalina e não contempladas nos cálculos dos sistemas isolados. Para todos os análogos, as geometrias obtidas foram completamente planas, o que é uma característica desejável para moléculas que agem como intercaladoras no processo de ação antitumoral. É também importante ressaltar que as estruturas dos diferentes análogos são similares, mostrando que os parâmetros geométricos (comprimentos e ângulos de ligação) não devem ser úteis para a distinção entre derivados ativos e inativos. No presente estudo, parâmetros geométricos específicos não foram considerados como descritores moleculares na análise QSAR, sendo utilizados apenas parâmetros estéreos globais, como área e volume molecular. A importância dos descritores estéreos, eletrônicos e termodinâmicos para a potência biológica das elipticinas é discutida no final desta seção.

\section{Propriedades das elipticinas em solução: energias de solvatação}

A densidade $(\rho)$ e o volume molar $\left(\mathrm{V}_{\mathrm{m}}\right)$ das misturas elipticinas/ solvente (água ou $\mathrm{CCl}_{4}$ ) foram calculados nas simulações de Monte Carlo. Como se tratam de soluções diluídas, os valores dessas propriedades devem ser próximos daqueles obtidos experimentalmente para os solventes puros. Os valores médios considerando todos os análogos foram $\rho=1,0104 \mathrm{~g} \mathrm{~cm}^{-3} \mathrm{e} \mathrm{V}_{\mathrm{m}}=17,93 \mathrm{~cm}^{3} \mathrm{~mol}^{-1}$ (água) e $\rho=1,5736 \mathrm{~g} \mathrm{~cm}^{-3}$ e V=97,83 $\mathrm{cm}^{3} \mathrm{~mol}^{-1}\left(\mathrm{CCl}_{4}\right)$. A densidade da água pura a $25^{\circ} \mathrm{C}$ é $0,997 \mathrm{~g} \mathrm{~cm}^{-3}$, e o volume molar igual a $17,99 \mathrm{~cm}^{3} \mathrm{~mol}^{-1}$. Para o $\mathrm{CCl}_{4}$ a $25^{\circ} \mathrm{C}, \rho=1,594 \mathrm{~g} \mathrm{~cm}^{-3}$ e V $_{\mathrm{m}}=97,73 \mathrm{~cm}^{3} \mathrm{~mol}^{-1}{ }^{17}$. Os resultados das densidades e volumes molares das soluções apresentam um desvio máximo de $1,6 \%$ para a densidade e $2 \%$ para o volume molar, mostrando a excelente qualidade da simulação de Monte Carlo para os estudos dos processos de solvatação das moléculas em ambos os solventes. A qualidade das simulações é também evidenciada na energia de interação solvente-solvente calculada pelo programa DIADORIM. Considerando todos os análogos apresentados na Tabela 1, a energia média de interação água-água foi igual a $-10,132 \mathrm{kcal} \mathrm{mol}^{-1}$ e $\mathrm{CCl}_{4}-\mathrm{CCl}_{4}$ igual a $-7,162 \mathrm{kcal} \mathrm{mol}^{-1}$. Valores típicos da energia de interação $\mathrm{H}_{2} \mathrm{O}-\mathrm{H}_{2} \mathrm{O}\left(\mathrm{E}_{\mathrm{AA}}\right)$ e $\mathrm{CCl}_{4}-\mathrm{CCl}_{4}\left(\mathrm{E}_{\mathrm{TT}}\right)$ encontrados na literatura ${ }^{17}$ situam-se em torno de -10 e $-7 \mathrm{kcal} \mathrm{mol}^{-1}$, respectivamente.

Na Tabela 2 são relatadas as energias de solvatação $\left(\Delta \mathrm{E}_{\mathrm{SOLV}}\right)$ calculadas nas simulações de Monte Carlo (MC) a $25^{\circ} \mathrm{C}$, as quais são comparadas às energias livres de solvatação $\left(\Delta \mathrm{G}_{\mathrm{SOLV}}\right)$ total e eletrostática, calculadas utilizando o modelo contínuo $\mathrm{PCM}^{7,8}$. Analisando os resultados obtidos da simulação de MC, pode-se observar claramente que a energia de hidratação das moléculas com carga líquida $+1\left(\sim-130 \mathrm{kcal} \mathrm{mol}^{-1}\right)$ é significativamente maior, em termos absolutos, que a energia de hidratação das moléculas neutras $\left(\sim-50 \mathrm{kcal} \mathrm{mol}^{-1}\right)$. Esta diferença não é observada em $\mathrm{CCl}_{4}$, sendo os valores de $\Delta \mathrm{E}_{\mathrm{Solv}}$ próximos de $-45 \mathrm{kcal} \mathrm{mol}^{-1}$. Do ponto de vista de forças intermoleculares, este comportamento distinto pode ser en- 
Tabela 2. Energias de solvatação (em kcal mol$\left.{ }^{-1}\right)$ das elipticinas em água e em $\mathrm{CCl}_{4}$ calculadas utilizando o método de $\mathrm{Monte}$ Carlo $\left(\Delta \mathrm{E}_{\mathrm{solv}}\right)$ e o modelo contínuo $\operatorname{PCM}\left(\Delta \mathrm{G}_{\text {SOLV }}\right)$

\begin{tabular}{|c|c|c|c|c|c|c|c|}
\hline & \multirow[b]{3}{*}{ carga } & \multicolumn{2}{|c|}{ Monte Carlo } & \multicolumn{4}{|c|}{$P C M$} \\
\hline & & \multirow[t]{2}{*}{$\Delta E_{S O L V}\left(H_{2} \mathrm{O}\right)$} & \multirow[t]{2}{*}{$\Delta E_{S O L V}\left(C C l_{4}\right)$} & \multicolumn{2}{|c|}{$\Delta G_{S O L V}\left(H_{2} \mathrm{O}\right)$} & \multicolumn{2}{|c|}{$\Delta G_{S O L V}\left(C C l_{4}\right)$} \\
\hline & & & & Total & Elet. $^{\mathrm{a}}$ & Total & Elet. ${ }^{\mathrm{a}}$ \\
\hline E01 & 0 & $-45,87 \pm 0,26$ & $-42,27 \pm 0,21$ & $-7,95$ & $-11,02$ & $-0,66$ & $-4,25$ \\
\hline E02 & 0 & $-50,61 \pm 0,31$ & $-43,74 \pm 0,22$ & $-11,42$ & $-14,70$ & $-1,26$ & $-5,69$ \\
\hline E03 & 0 & $-51,79 \pm 0,31$ & $-43,75 \pm 0,31$ & $-13,20$ & $-16,32$ & $-1,65$ & $-6,31$ \\
\hline E04 & 0 & $-49,49 \pm 0,30$ & $-42,68 \pm 0,21$ & $-11,71$ & $-14,88$ & $-1,21$ & $-5,86$ \\
\hline E05 & 0 & $-48,50 \pm 0,29$ & $-45,84 \pm 0,25$ & $-9,48$ & $-12,82$ & $-0,76$ & $-5,06$ \\
\hline E06 & +1 & $-129,76 \pm 0,60$ & $-43,30 \pm 0,23$ & $-41,82$ & $-45,29$ & $-18,15$ & $-23,50$ \\
\hline E07 & +1 & $-137,65 \pm 0,63$ & $-46,07 \pm 0,23$ & $-47,48$ & $-50,82$ & $-19,15$ & $-25,62$ \\
\hline E08 & +1 & $-128,66 \pm 0,58$ & $-47,23 \pm 0,22$ & $-40,74$ & $-44,32$ & $-17,71$ & $-23,08$ \\
\hline E09 & +1 & $-130,88 \pm 0,60$ & $-46,14 \pm 0,23$ & $-38,69$ & $-42,38$ & $-17,20$ & $-22,30$ \\
\hline E10 & +1 & $-131,64 \pm 0,58$ & $-47,63 \pm 0,22$ & $-44,23$ & $-47,79$ & $-18,19$ & $-24,44$ \\
\hline
\end{tabular}

${ }^{a}$ Contribuição eletrostática para a energia de solvatação.

tendido em função da presença de interações carga-dipolo entre os sítios do soluto e da molécula de água, enquanto que em tetracloreto de carbono as interações de van der Waals são predominantes.

Os resultados PCM (Tabela 2) mostram uma solvatação mais favorável em água que em $\mathrm{CCl}_{4}$ tanto para as moléculas neutras (E01 a E05) quanto para as moléculas com carga líquida +1 (E06 a E10). Neste nível de teoria, as energias de solvatação das moléculas carregadas são mais pronunciadas que dos compostos neutros em água e em $\mathrm{CCl}_{4}$. Analisando os dados da Tabela 2 e as Figuras $2 \mathrm{a}$ e $2 \mathrm{~b}$ pode ser observada uma correlação linear satisfatória entre os valores de $\Delta \mathrm{E}_{\text {SOLV }}$ (clássico) e $\Delta \mathrm{G}_{\mathrm{SOLV}}$ (quântico) para solução aquosa, para o conjunto de moléculas neutras, $r=0,961$ (Figura 2a) e para as moléculas carregadas $\mathrm{r}=0,830$ (Figura $2 \mathrm{~b}$ ). $\mathrm{Em} \mathrm{CCl}_{4}$ nenhuma correlação entre os valores clássicos e quânticos foi observada (ver Figuras $3 \mathrm{a}$ e $3 b$ ). Esse resultado reflete a natureza da parametrização do modelo
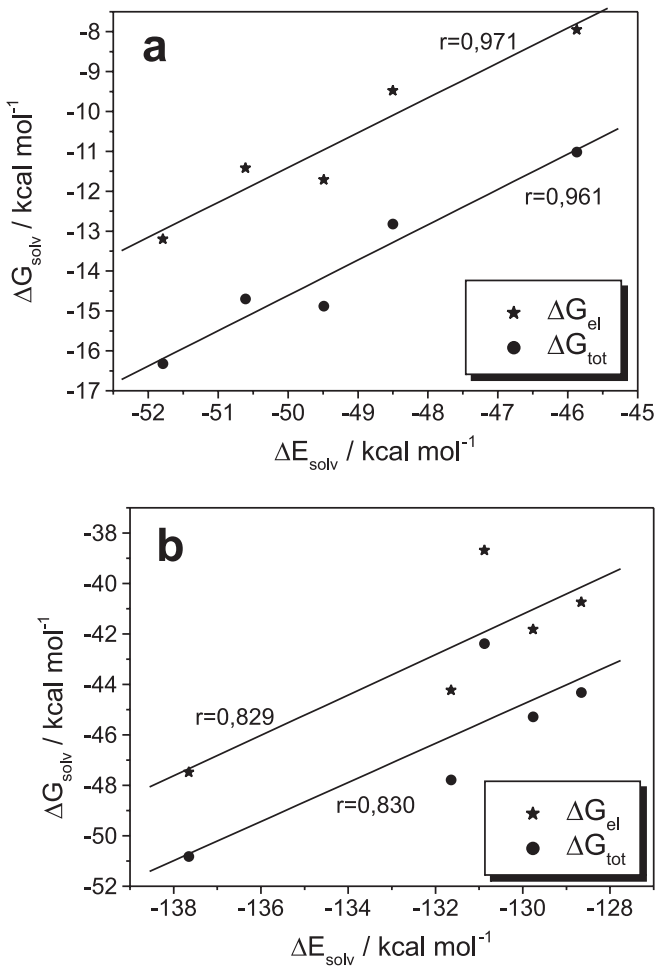

Figura 2. Correlação entre $\Delta E_{S O L V}$ e $\Delta G_{\text {SOLV }}$ em água: (a) moléculas neutras e (b) moléculas com carga líquida +1
PCM, a qual foi desenvolvida principalmente para descrever energias de hidratação $0^{7,8}$.

\section{Propriedades das elipticinas em solução: análise estrutural}

O primeiro grupo de funções de correlação $(\mathrm{g}(\mathrm{r}))$ analisado envolveu o átomo de nitrogênio piridínico $\left(\mathrm{N}_{2}\right)$ e os sítios $\mathrm{O}_{\mathrm{w}}$ e $\mathrm{H}_{\mathrm{w}}$ da molécula de água. Essas funções foram designadas como $\mathrm{g}\left[\mathrm{N}_{2} \mathrm{H}_{\mathrm{w}}\right](\mathrm{r})$ e $\mathrm{g}\left[\mathrm{N}_{2} \mathrm{O}_{\mathrm{w}}\right](\mathrm{r})$ e mostram o grau de organização das moléculas de água em torno do sítio $\mathrm{N}_{2}$, o qual é distinto para as subclasses de moléculas neutras (Figuras 4a e 4b) e catiônicas (Figuras 4c e 4d). O perfil dessas funções é semelhante nas moléculas E01 a E05, que são os derivados neutros da elipticina. Isso pode ser explicado pelo fato da carga parcial sobre o átomo $\mathrm{N}_{2}$ ser a mesma em todas as moléculas. Na Tabela 3, a carga parcial, a posição do primeiro máximo local e
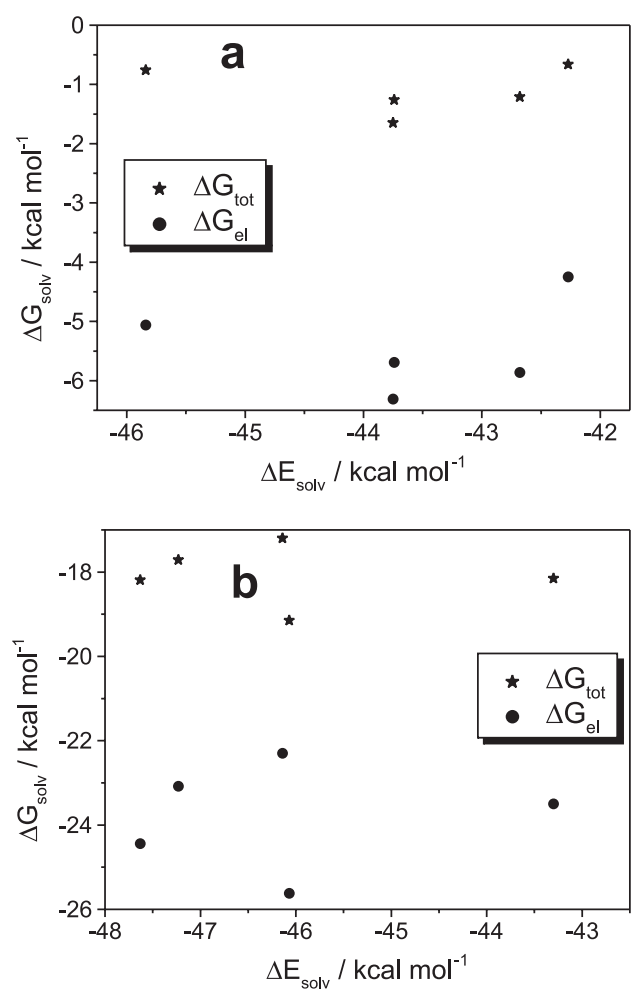

Figura 3. Correlação entre $\Delta E_{\text {SOLV }}$ e $\Delta G_{\text {SOLV }}$ em $C C l_{4}$ : (a) moléculas neutras e (b) moléculas com carga líquida +1 
Tabela 3. Descrição dos primeiros picos de $\mathrm{g}(\mathrm{r})$ para os pares $\mathrm{N}_{2}-\mathrm{H}_{\mathrm{w}}$ e $\mathrm{N}_{2}-\mathrm{O}_{\mathrm{w}}$

\begin{tabular}{|c|c|c|c|c|c|c|c|}
\hline & \multirow[b]{2}{*}{$\mathrm{Q}\left(\mathrm{N}_{2}\right)$} & \multicolumn{3}{|c|}{$g\left[N_{2} H_{w}\right](r)$} & \multicolumn{3}{|c|}{$g\left[\mathrm{~N}_{2} \mathrm{O}_{w}\right](r)$} \\
\hline & & r(1ºmáx)/ Å & $\mathrm{r}\left(1^{\circ} \mathrm{omin}\right) / \AA$ & $\mathrm{NC}^{\mathrm{a}}$ & r(1ํomáx)/ Å & $\mathrm{r}(1 \stackrel{0}{\mathrm{o}} \mathrm{min}) / \AA$ & $\mathrm{NC}^{\mathrm{a}}$ \\
\hline E01 & $-0,68$ & 1,9 & 2,7 & 1,9 & 2,9 & 3,3 & 2,0 \\
\hline E02 & $-0,68$ & 1,9 & 2,7 & 1,8 & 2,9 & 3,3 & 2,1 \\
\hline E03 & $-0,68$ & 1,9 & 2,5 & 1,1 & 2,9 & 3,1 & 1,1 \\
\hline E04 & $-0,68$ & 1,9 & 2,7 & 1,6 & 2,9 & 3,3 & 1,6 \\
\hline E05 & $-0,68$ & 1,9 & 2,7 & 1,3 & 2,9 & 3,3 & 1,4 \\
\hline
\end{tabular}

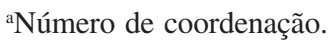
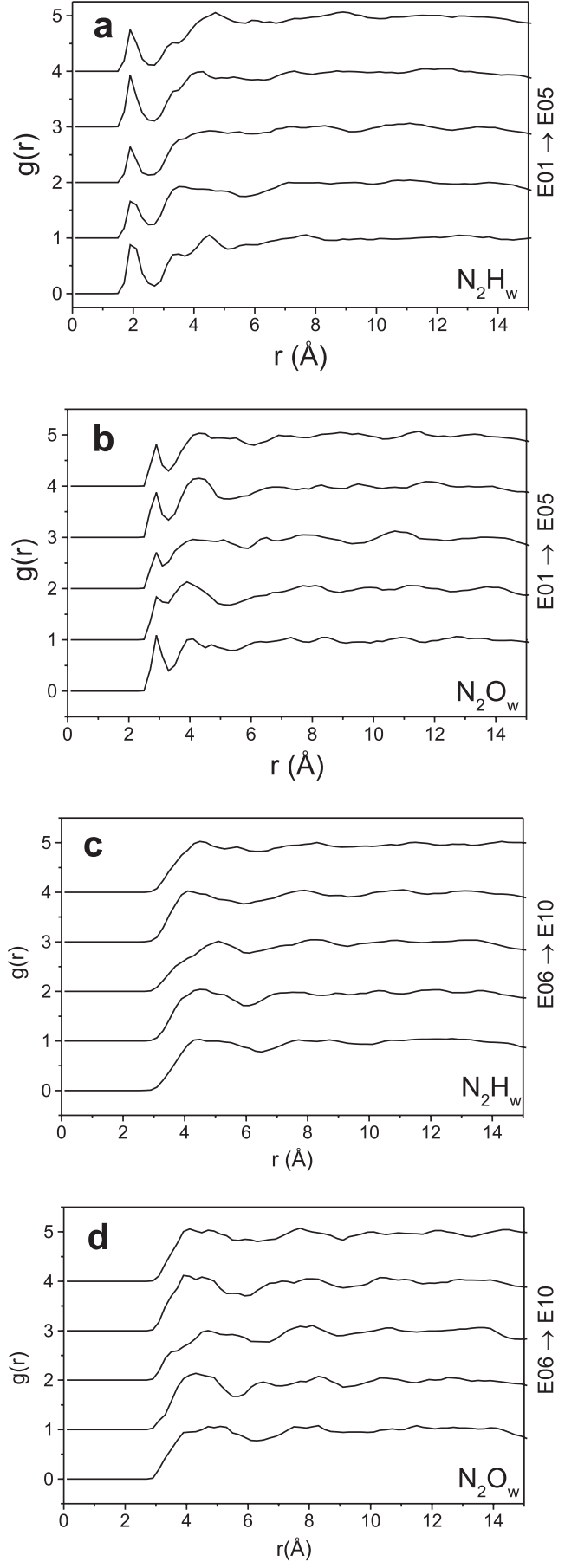

Figura 4. Comparação entre as funções de distribuição radial de pares dos derivados da elipticina: (a) correlações $N_{2}-H_{w}$; (b) correlações $N_{2}-O_{w}$; (c) correlações $N_{2}-H_{w}$ e (d) correlações $N_{2}-O_{w}$ do primeiro mínimo local e a respectiva integral, que fornece o número de coordenação (NC) do solvente em torno deste sítio do soluto, são relatadas para as moléculas E01 a E05. De acordo com os dados da Tabela 3, pode ser observado que a relação entre os números de coordenação de $\mathrm{g}\left[\mathrm{N}_{2} \mathrm{H}_{\mathrm{w}}\right](\mathrm{r})$ e de $\mathrm{g}\left[\mathrm{N}_{2} \mathrm{O}_{\mathrm{w}}\right](\mathrm{r})$ mostra que a razão entre o número de hidrogênios $\left(\mathrm{H}_{\mathrm{w}}\right)$ e oxigênio $\left(\mathrm{O}_{\mathrm{w}}\right)$ da água vizinhos ao sítio $\mathrm{N}_{2}$ é aproximadamente igual a 1. Em uma primeira análise podemos afirmar, com base nas Figuras $4 \mathrm{a}$ e 4b, que há um certo grau de organização da água na região do sítio $\mathrm{N}_{2}$, pois há um pico bem definido em $\mathrm{g}(\mathrm{r})$, mas não podemos concluir que essa organização é devida à formação de ligações de hidrogênio, pois o perfil das funções de correlação $\mathrm{N}_{2}-\mathrm{H}_{\mathrm{w}}$ não apresenta o segundo pico bem definido característico deste tipo de interação. Analisando as mesmas funções de distribuição radial de pares para as moléculas com carga líquida +1 (E06 a E10) (Figuras 4c e 4d), pode ser notado que toda a estruturação da água é perdida, sendo o primeiro pico centrado a uma distância média de cerca de 4 Å.

Para investigar a estruturação local dos solventes em torno dos sítios $\mathrm{C}_{7}, \mathrm{C}_{8}$ e $\mathrm{C}_{9}$, os quais apresentam características específicas dentro da série de derivados analisados, foram calculadas as funções de correlação entre estes sítios $\left(\mathrm{C}_{7}\right.$ a $\left.\mathrm{C}_{9}\right)$ e os sítios da molécula de água. Estas funções foram analisadas para as dez moléculas, sendo encontrada muita semelhança entre elas na primeira camada de solvatação. Na Figura 5 é mostrada a função g(r) apenas para o sítio $\mathrm{C}_{7}$ e o oxigênio da água $\left(\mathrm{O}_{\mathrm{w}}\right)$. $\mathrm{O}$ que pode ser observado para todas as moléculas é que a água se distribui de maneira semelhante para os diferentes análogos nessa região das elipticinas, como mostra o primeiro pico de $\mathrm{g}(\mathrm{r})$ centrado em cerca de $3,7 \AA$.

Uma correlação importante encontrada neste estudo foi entre o hidrogênio do anel pirrol $\left(\mathrm{H}_{6}\right)$ e a água, mostrada nas Figuras $6 \mathrm{a}$ e $6 \mathrm{~b}$. As funções $\mathrm{g}\left[\mathrm{H}_{6} \mathrm{O}_{\mathrm{w}}\right](\mathrm{r})$ e $\mathrm{g}\left[\mathrm{H}_{6} \mathrm{H}_{\mathrm{w}}\right](\mathrm{r})$ foram analisadas para caracterizar a presença de ligações de hidrogênio nesse sítio. Nas Figuras 6a e 6b são mostrados apenas os gráficos dessas funções para as moléculas E01 a E08, que possuem um hidrogênio pirrólico. As moléculas E09 e E10 possuem um grupo metila nessa posição. O primeiro máximo das

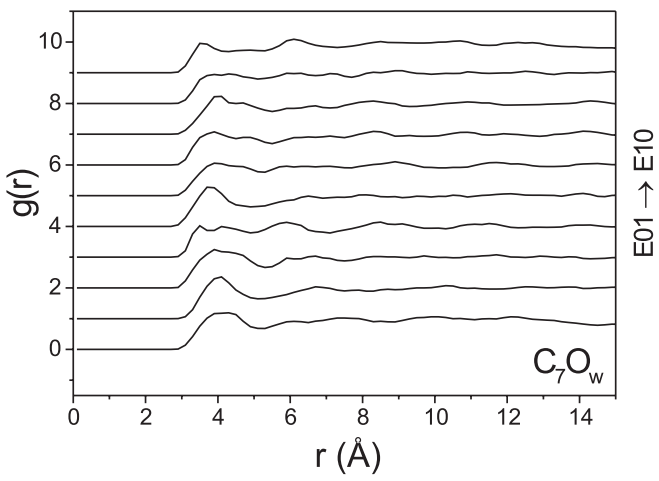

Figura 5. Funções de distribuição radial calculadas para as elipticinas em água considerando o par de átomos $C_{7}-O_{w}$ 
funções $\mathrm{g}\left[\mathrm{H}_{6} \mathrm{O}_{\mathrm{w}}\right](\mathrm{r})$ em todas as moléculas (E01 a E08) está em 1,9 $\mathrm{A}$ e o primeiro máximo das funções $\mathrm{g}\left[\mathrm{H}_{6} \mathrm{O}_{\mathrm{w}}\right](\mathrm{r})$ está em $2,5 \AA$ A . Na Tabela 4 está a descrição dos primeiros picos dessas duas funções. Esses resultados mostram que há uma organização das moléculas de água que é típica de ligações de hidrogênio, e como o número de coordenação entre $\mathrm{H}_{6}$ e $\mathrm{O}_{\mathrm{w}}$ é cerca de 1, podemos afirmar que há em média uma ligação de hidrogênio com a água nesse sítio.

Para avaliar a estruturação das elipticinas em meio lipofílico, calculamos as funções de distribuição radial de pares dessas moléculas solvatadas em $\mathrm{CCl}_{4}$, considerado um solvente adequado para representar o meio lipofílico em simulação computacional. As funções de distribuição radial de pares entre diferentes sítios das elipticinas e o centro de massa do $\mathrm{CCl}_{4}$ foram analisadas. A Figura 7 mostra o grupo de funções envolvendo os sítios $\mathrm{N}_{2}$ e $\mathrm{C}_{7}$ das elipticinas e o átomo de $\mathrm{C}$ do $\mathrm{CCl}_{4}$. Para todas as moléculas de soluto na região do sítio $\mathrm{N}_{2}, \mathrm{O} \mathrm{CCl}_{4}$ se distribui da mesma maneira, como fica evidente pelo comportamento de g(r) (Figura 7a). Observando-se a Figura 7b pode-se ver que a distância entre os centros de massa das moléculas do solvente $\mathrm{CCl}_{4}$, representados pelos átomos de carbono, fica a cerca de $5 \AA$ do sítio $\mathrm{C}_{7}$ dos solutos E01 a E10. Resultados similares
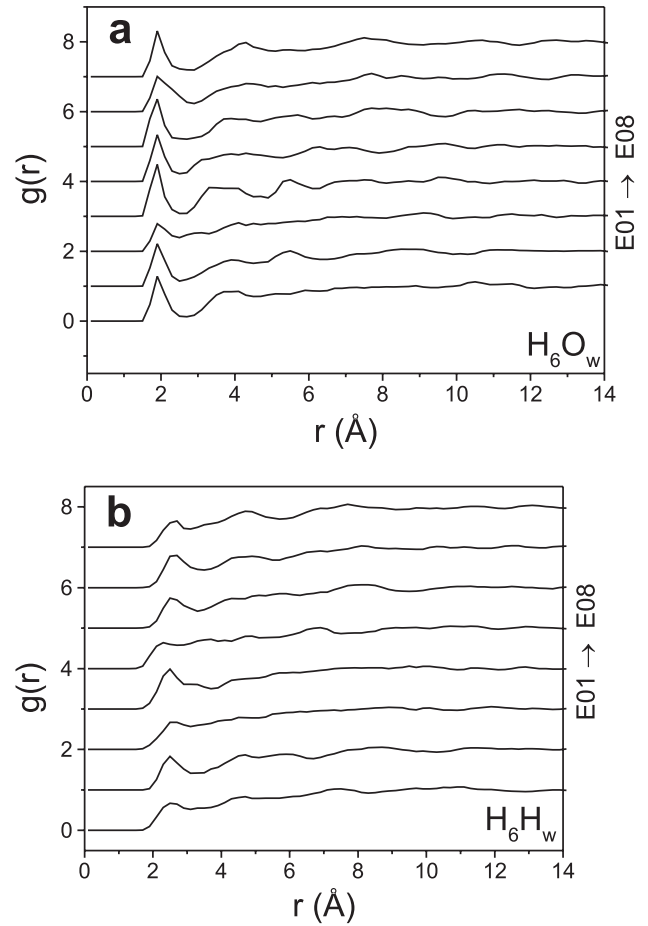

Figura 6. Funções de distribuição radial de pares das elipticinas em relação aos sítios da molécula de água: (a) $H_{6}-O_{w} e$ (b) $H_{6}-H_{w}$ foram obtidos considerando os átomos $\mathrm{C}_{8}$ e $\mathrm{C}_{9}$ das elipticinas. Este resultado mostra que o $\mathrm{CCl}_{4}$, ao contrário da água, mantém uma distância constante das elipticinas, sem apresentar uma estruturação local de importância.

\section{Análise quantitativa de relação estrutura-atividade (QSAR) envolvendo elipticinas}

Os valores dos descritores moleculares calculados e parâmetros biológicos obtidos da literatura para a série de elipticinas selecionadas são apresentados na Tabela 5. Os modelos QSAR desenvolvidos envolveram a constante de associação com o DNA (Kapp). De acordo com os trabalhos de Auclair ${ }^{3}$, as elipticinas interagem in vitro com o DNA através de um processo de intercalação com Kapp maior que $10^{5} \mathrm{M}^{-1}$. O desenvolvimento dos modelos foi feito considerando uma busca sistemática de correlações do parâmetro biológico (logKapp) com uma e duas variáveis independentes. É importante ressaltar que o número reduzido de compostos considerados no presente trabalho limita os modelos desenvolvidos ao uso de poucos descritores moleculares ${ }^{18-22}$.
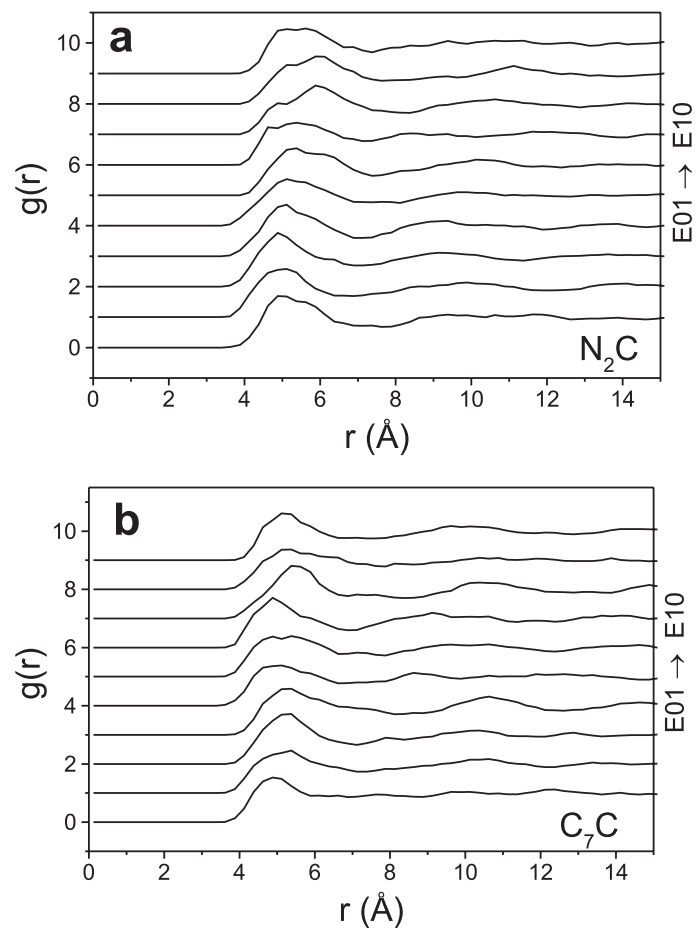

Figura 7. Funções de distribuição radial de pares entre os sítios $\mathrm{N}_{2}$ e $\mathrm{C}_{7}$ das elipticinas e o centro de massa do $\mathrm{CCl}_{4}$ : (a) $N_{2}-C$; (b) $C_{7}-C$

Tabela 4. Descrição dos primeiros picos de $\mathrm{g}(\mathrm{r})$ para os pares $\mathrm{H}_{6}-\mathrm{H}_{\mathrm{w}}$ e $\mathrm{H}_{6}-\mathrm{O}_{\mathrm{w}}$

\begin{tabular}{|c|c|c|c|c|c|c|c|}
\hline & \multirow[b]{2}{*}{$\mathrm{Q}\left(\mathrm{H}_{6}\right)$} & \multicolumn{3}{|c|}{$g\left[H_{6} H_{w}\right](r)$} & \multicolumn{3}{|c|}{$g\left[H_{6} O_{w}\right](r)$} \\
\hline & & r(1-máx)/ Å & $\mathrm{r}(1-\mathrm{min}) / \AA$ & $\mathrm{NC}^{\mathrm{a}}$ & r(1@máx)/ Å & $\mathrm{r}(1-\mathrm{min}) / \AA$ & $\mathrm{NC}^{\mathrm{a}}$ \\
\hline E01 & 0,44 & 2,5 & 3,1 & 3,9 & 1,9 & 2,9 & 1,2 \\
\hline E02 & 0,41 & 2,5 & 3,3 & 4,4 & 1,9 & 2,5 & 1,0 \\
\hline E03 & 0,40 & 2,5 & 3,3 & 4,1 & 1,9 & 2,7 & 1,2 \\
\hline E04 & 0,43 & 2,5 & 2,9 & 4,0 & 1,9 & 2,7 & 1,0 \\
\hline E05 & 0,42 & 2,3 & 2,9 & 3,3 & 1,9 & 2,5 & 1,1 \\
\hline E06 & 0,45 & 2,5 & 3,1 & 3,8 & 1,9 & 2,5 & 1,1 \\
\hline E07 & 0,43 & 2,5 & 3,5 & 3,8 & 1,9 & 2,7 & 1,0 \\
\hline E08 & 0,45 & 2,7 & 3,1 & 3,3 & 1,9 & 2,9 & 1,3 \\
\hline
\end{tabular}

${ }^{a}$ Número de coordenação. 
Tabela 5. Propriedades eletrônicas calculadas para os derivados da elipticina no nível HF/6-31G(d): Cargas atômicas ChelpG (Q/a.u.), momento de dipolo elétrico $(\mu / \mathrm{D})$, energias dos orbitais moleculares de fronteira (E/hartree). Os descritores estéreos área $\left(\mathrm{em} \AA^{2}\right.$ ) e volume $\left(\mathrm{em} \AA^{3}\right.$ ) foram calculados utilizando as geometrias $a b$ initio

\begin{tabular}{|c|c|c|c|c|c|c|c|c|c|c|}
\hline & $\mathrm{Q}\left(\mathrm{N}_{2}\right)$ & $\mathrm{Q}\left(\mathrm{N}_{6}\right)$ & $\mathrm{Q}\left(\mathrm{C}_{7}\right)$ & $\mathrm{Q}\left(\mathrm{C}_{9}\right)$ & $\mu$ & $\mathrm{E}_{\text {номо }}$ & $\mathrm{E}_{\text {LUMO }}$ & area & volume & $\log \mathrm{K}_{\text {app }}{ }^{a}$ \\
\hline E01 & $-0,68$ & $-0,65$ & $-0,28$ & $-0,17$ & 3,9 & $-0,261$ & 0,080 & 263,92 & 234,35 & $-0,824$ \\
\hline E02 & $-0,68$ & $-0,61$ & $-0,20$ & 0,48 & 4,2 & $-0,252$ & 0,079 & 279,37 & 246,87 & \\
\hline E03 & $-0,68$ & $-0,56$ & $-0,21$ & 0,45 & 2,5 & $-0,258$ & 0,075 & 274,35 & 243,07 & 0,301 \\
\hline E04 & $-0,68$ & $-0,51$ & 0,34 & $-0,09$ & 4,8 & $-0,259$ & 0,080 & 274,32 & 242,99 & \\
\hline E05 & $-0,68$ & $-0,60$ & $-0,23$ & 0,42 & 5,1 & $-0,256$ & 0,079 & 294,99 & 260,17 & \\
\hline E06 & 0,14 & $-0,66$ & $-0,29$ & $-0,17$ & 7,9 & $-0,388$ & $-0,071$ & 287,54 & 253,47 & $-0,638$ \\
\hline E07 & 0,12 & $-0,56$ & $-0,15$ & 0,45 & 8,1 & $-0,375$ & $-0,069$ & 298,11 & 262,08 & 0,041 \\
\hline E08 & $-0,02$ & $-0,68$ & $-0,31$ & $-0,17$ & 7,7 & $-0,384$ & $-0,070$ & 300,83 & 269,50 & \\
\hline E09 & 0,09 & $-0,21$ & $-0,23$ & $-0,15$ & 8,1 & $-0,383$ & $-0,072$ & 302,94 & 270,19 & 0,532 \\
\hline E10 & 0,10 & $-0,11$ & $-0,08$ & 0,47 & 8,2 & $-0,372$ & $-0,068$ & 313,65 & 278,78 & 1,146 \\
\hline
\end{tabular}

${ }^{a}$ Constante de associação obtida da ref. 3 .

Considerando apenas um descritor molecular, o melhor modelo QSAR obtido é apresentado na Equação (3).

$\operatorname{logKapp}=+2,79( \pm 1,78) \mathrm{Q}\left(\mathrm{N}_{6}\right)+1,37( \pm 0,90)$ $\left(\mathrm{n}=6 ; \mathrm{r}=0,895 ; \mathrm{s}=0,368 ; \mathrm{F}=16,136 ; \mathrm{Q}^{2}=0,620 ; \mathrm{SPRESS}=0,508\right)$

O modelo da Equação (3) mostra que quanto menor a densidade de cargas no átomo $\mathrm{N}_{6}$ maior a constante de associação Kapp. $\mathrm{O}$ gráfico da Figura 8a mostra os valores preditos pelo modelo (3) e aqueles observados. Outros modelos com menor significância estatística foram obtidos considerando $\mathrm{Q}\left(\mathrm{C}_{7}\right)$ e $\Delta \mathrm{E}_{\mathrm{SOLV}}\left(\mathrm{CCl}_{4}\right)$. Apesar da perda da significância estatística, modelos com duas variáveis independentes foram também analisados. Os melhores modelos foram obtidos considerando o momento de dipolo elétrico $(\mu)$ e o volume (V) ou área (A) molecular (Equações 4 e 5).

$\operatorname{logKapp}=-0,31( \pm 0,05) \mu+0,07( \pm 0,01) \mathrm{V}-16,93( \pm 1.64)$

$\left(n=6 ; r=0,998 ; s=0,056 ; F=433,664 ; Q^{2}=0,989 ;\right.$ SPRESS $\left.=0,101\right)$

$\operatorname{logKapp}=-0,34( \pm 0,07) \mu+0,07( \pm 0,01) \mathrm{A}-18,15( \pm 2,56)$

$\left(\mathrm{n}=6 ; \mathrm{r}=0,996 ; \mathrm{s}=0,081 ; \mathrm{F}=206,431 ; \mathrm{Q}^{2}=0,969 ;\right.$ SPRESS $\left.=0,168\right)$

As Equações (4) e (5) mostram que a diminuição da polaridade e o aumento da área ou volume molecular favorecem o processo de intercalação. Outro modelo também significativo do ponto de vista estatístico e relevante na análise do sistema em questão foi obtido considerando as cargas atômicas nos sítios $\mathrm{N}_{6}$ e $\mathrm{C}_{9}$ (Equação 6).

$\operatorname{logKapp}=+2,45( \pm 0,92) \mathrm{Q}\left(\mathrm{N}_{6}\right)+0,92( \pm 0,64) \mathrm{Q}\left(\mathrm{C}_{9}\right)+1,08( \pm 0,49)(6)$ $\left(\mathrm{n}=6 ; \mathrm{r}=0,984 ; \mathrm{s}=0,169 ; \mathrm{F}=45,976 ; \mathrm{Q}^{2}=0,863 ; \mathrm{SPRESS}=0,353\right)$

No modelo da Equação (6), a diminuição da densidade de cargas no átomo $\mathrm{N}_{6}$ e a presença de substituintes retiradores de elétrons em $\mathrm{C}_{9}$ levam a um aumento na constante de associação com o DNA. A comparação entre os valores observados e previstos pelos modelos (4) e (6) é representada nas Figuras $8 b$ e $8 c$, respectivamente.

É importante correlacionar a análise quantitativa representada pelas equações (3-6) com trabalhos anteriores envolvendo análises qualitativas entre estrutura e atividade de elipticinas. De acordo com os trabalhos de Auclair ${ }^{3}$, a hidroxilação da posição $\mathrm{C}_{9}$ é uma modificação estrutural importante para aumentar Kapp, sendo esse efeito relacionado à possibilidade de formação de ligação de hidrogênio das elipticinas com os grupos fosfatos. Esta observação pode ser quantitativamente descrita pela diminuição na densidade eletrônica no átomo $\mathrm{C}_{9}$, de acordo com o modelo (6). Na análise qualitativa
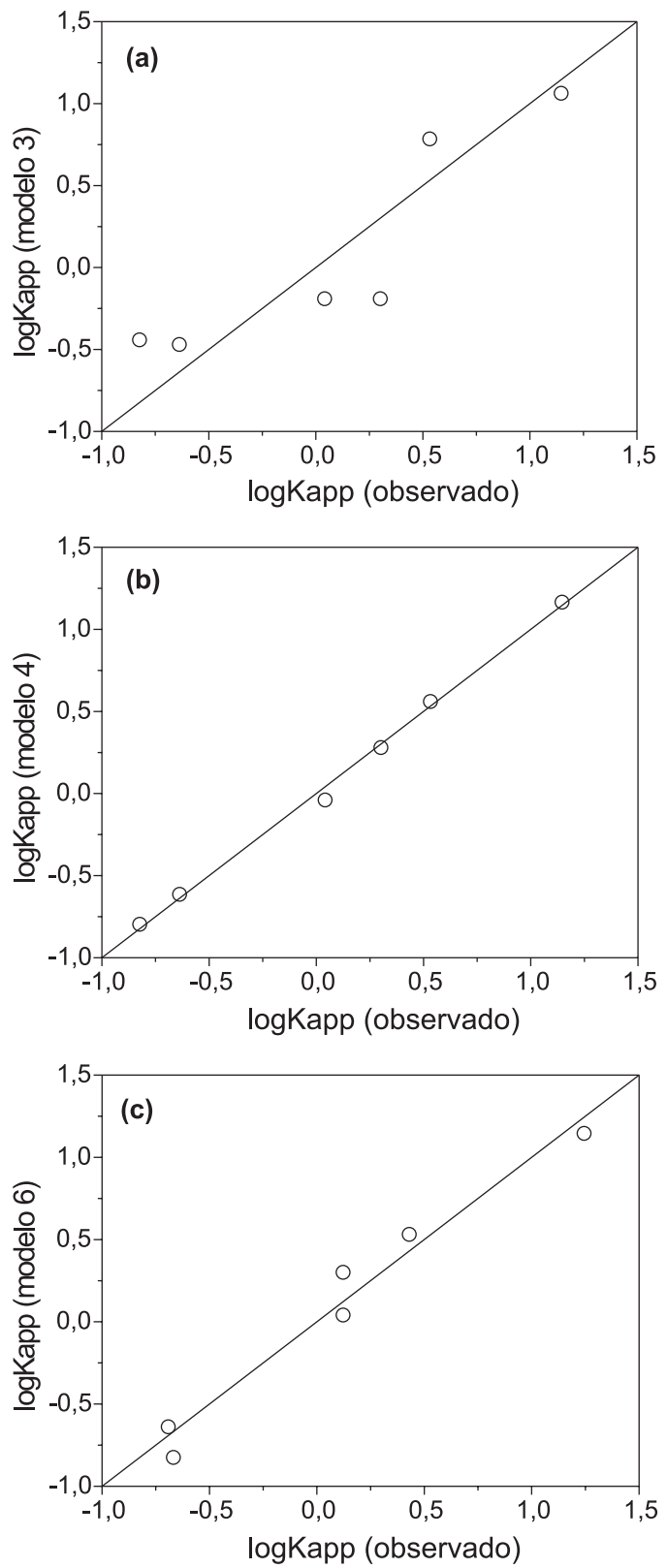

Figura 8. Correlação entre os valores de logKapp preditos pelos modelos (3), (4) e (6) e determinados experimentalmente 
desenvolvida por Dantas e colaboradores ${ }^{23}$, um baixo efeito citotóxico foi correlacionado com a alta densidade de elétrons em $\mathrm{N}_{6}$, sendo esse efeito quantitativamente descrito pelos modelos (3) e (6). Neste mesmo estudo ${ }^{23}$ e também no trabalho de Barone e colaboradores ${ }^{24}$, o momento de dipolo elétrico mostrou-se relevante para a atividade antitumoral das elipticinas. Esse fato é descrito pelos modelos (4) e (5), os quais mostram também a importância do volume molecular para o processo de intercalação. Esta evidência pode ser relacionada com os resultados de Devraj e Cushman ${ }^{4}$, os quais demonstraram a importância de grupos volumosos no anel carbazol para a potência biológica desta classe de moléculas.

Finalmente, é importante ressaltar que a constante de associação Kapp é um parâmetro relevante para a descrição dos efeitos citotóxicos das elipticinas ${ }^{3,23,24}$, entretanto, alguns compostos inativos apresentam altos valores de Kapp. Isto sugere a necessidade de uma investigação mais profunda incluindo outros descritores moleculares e parâmetros biológicos na análise QSAR.

\section{CONCLUSÃO}

No presente trabalho a estrutura e as propriedades moleculares de diferentes análogos da elipticina foram calculadas considerando métodos quanto-mecânicos $a b$ initio e simulação de Monte Carlo. Todas as estruturas otimizadas apresentaram conformações completamente planas, o que constitui um requisito importante para o modo de ação antitumoral de agentes intercaladores. Em solução aquosa, uma boa correlação foi obtida entre os valores de $\Delta \mathrm{G}_{\text {SOLV }}$ calculados pelo modelo contínuo PCM-UAHF e de $\Delta \mathrm{E}_{\mathrm{Solv}}$ obtidos das simulações de Monte Carlo, sendo mais significativa para o conjunto de moléculas neutras $(\mathrm{r}=0,961)$. Este resultado é importante no sentido de que modelos contínuos de solvatação possuem um custo computacional relativamente baixo, além de manterem o formalismo teórico da mecânica quântica e, portanto, representam uma alternativa para o cálculo de energias de solvatação relativas em processos químicos. $\mathrm{Em} \mathrm{CCl}_{4}$ nenhuma correlação foi obtida entre os valores de $\Delta \mathrm{G}_{\mathrm{SOLV}} \mathrm{e}$ $\Delta \mathrm{E}_{\mathrm{SOLV}}$. Modelos de relação quantitativa entre estrutura e atividade (QSAR) foram analisados considerando parâmetros eletrônicos (cargas atômicas), estéreos (volume e área molecular) e termodinâmicos (energias de solvatação) como variáveis independentes e a constante de associação com o DNA (logKapp) como descritor biológico. De forma geral, os modelos obtidos mostraram a importância dos descritores eletrônicos para a interação das elipticinas com o DNA. A alquilação do átomo $\mathrm{N}_{6}$ e a presença de grupos retiradores de elétrons em $\mathrm{C}_{9}$ aumentam Kapp. Este mesmo efeito é previsto com a diminuição do momento de dipolo elétrico e o aumento do volume molecular. Estes resultados quantitativos estão em acordo com estudos de relação estrutura-atividade (SAR), teóricos e experimentais, relatados na literatura. Entretanto, é importante ressaltar que os modelos desenvolvidos no presente trabalho devem ser aprimorados, considerando um conjunto maior de compostos e descritores moleculares que contemplem outros efeitos como, por exemplo, a lipofilicidade. Estudos estão sendo desenvolvidos nesta direção e serão relatados oportunamente.

\section{AGRADECIMENTOS}

Os autores agradecem às agências financiadoras FAPEMIG, CNPq e FINEP pelo suporte financeiro e bolsas concedidas.

\section{REFERÊNCIAS}

1. Juret, P.; Heron, J. F.; Couette, J. E.; Delozier, T.; Letalaer, J. Y.; Cancer Treat. Rep. 1982, 66, 1909.

2. Sizum, P.; Auclair, C.; Lescot, E.; Paoletti, C.; Perly, B.; Fermandjian, S.; Biopolymers 1988, 27, 1096.

3. Auclair, C.; Arch. Biochem. Biophys. 1987, 259, 1.

4. Devraj, R.; Cushman, M.; Bioorg. Med. Chem. Lett. 1997, 7, 369.

5. Frisch, M. J.; Trucks, G. W.; Schlegel, H. B.; Scuseria, G. E.; Robb, M. A.; Cheeseman, J. R.; Zakrzewski, V. G.; Montgomery, Jr. J. Á.; Stratmann, R. E.; Burant, J. C.; Dapprich, S.; Millam, J. M.; Daniels, A. D.; Kudin, K. N.; Strain, M. C.; Farkas, O.; Tomasi, J.; Barone, V.; Cossi, M.; Cammi, R.; Mennucci, B.; Pomelli, C.; Adamo, C.; Clifford, S.; Ochterski, J.; Petersson, G. A.; Ayala, P. Y.; Cui, Q.; Morokuma, K.; Malick, D. K.; Rabuck, A. D.; Raghavachari, K.; Foresman, J. B.; Cioslowski, J.; Ortiz, J. V.; Stefanov, B. B.; Liu, G.; Liashenko, A.; Piskorz, P.; Komaromi, I.; Gomperts, R.; Martin, R. L.; Fox, D. J.; Keith, T.; Al-Laham, M. A.; Peng, C. Y.; Nanayakkara, A.; Gonzalez, C.; Challacombe, M.; Gill, P. M. W.; Johnson, B.; Chen, W.; Wong, M. W.; Andres, J. L.; Head-Gordon, M.; Replogle, E. S.; Pople, J. A.; Gaussian 98, Revision A.6.; Gaussian, Inc.: Pittsburgh PA, 1998.

6. PC Spartan Pro, Wavefunction, Inc., USA, 1999

7. Cossi, M.; Barone, V.; Cammi, R.; Tomasi, J.; Chem. Phys. Lett. 1996, 255, 327.

8. Barone, V.; Cossi, M.; Tomasi, J.; J. Chem. Phys. 1997, 107, 3210

9. DIADORIM.TPT; Freitas, L. C. G., Fortran Code, 1990.

10. Dos Santos, H. F.; Nascimento, C. S.; Belletato, P.; De Almeida, W. B.; J. Mol. Struct. (Theochem) 2003, 626, 305.

11. Jorgensen, W. L.; J. Chem. Phys. 1979, 71, 5034.

12. Mahoney, M. W.; Jorgensen, W. L.; J. Chem. Phys. 2000, 112, 8910.

13. BOSS Version 3.5: Jorgensen, W. L.; Biorganic and Organica Simulation System 1995.

14. De Oliveira, D. B.; Gaudio, A. C.; Quant. Struct.-Act. Relat. 2000, 19, 599.

15. Courseille, C.; Busetta, B.; Hospital, H.; Acta Crystallogr., Sect. B: Struct. Sci. 1974, 30, 2628.

16. Aggarwal, A.; Neidel, S.; Sinsbury, M.; Acta Crystallogr., Sect. C: Cryst. Struct. Commun. 1983, 39, 631.

17. Lide, D. R., ed., CRC Handbook of Chemistry and Physics, $81^{\text {st }}$ ed., 2001.

18. Topliss, J. G.; Costello, R. J.; J. Med. Chem. 1972, 15, 1066.

19. Unger, S. H.; Hansch, C.; J. Med. Chem. 1973, 16, 745.

20. Wold, S.; Quant. Struct.-Act. Relat. 1991, 10, 191.

21. Boyd, R. J.; Boyd, S. L.; J. Am. Chem. Soc. 1992, 114, 1652

22. Kubinyi, H. QSAR: Hansch Analysis and Related Approaches; VCH, 1993.

23. Dantas, S. O.; Lavarda, F. C.; Galvão, D. S.; Laks, B.; J. Mol. Struct. (Theochem) 1992, 253, 319

24. Barone, P. M. V. B.; Dantas, S. O.; Galvão, D. S.; J. Mol. Struct. (Theochem) 1999, 465, 219 